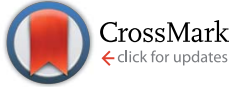

Cite this: RSC Adv., 2017, 7, 4430
Received 12th October 2016 Accepted 15th December 2016

DOI: 10.1039/c6ra25132c

www.rsc.org/advances

\section{Influences of structure and multi-intermolecular forces on rheological and oil displacement properties of polymer solutions in the presence of $\mathrm{Ca}^{2+} / \mathrm{Mg}^{2+}$}

\begin{abstract}
Wanfen Pu, Feng Jiang, Bing Wei, ${ }^{*}$ Yanli Tang and Yanyan He
Salinity-tolerance is one of the crucial factors determining the applicability of a polymer in Enhancing Oil Recovery (EOR) process. In the current study, the rheological and oil displacement properties of four types of polymers with similar structures, including a comb polymer (KYPAM), a double tailed hydrophobic associating polymer (DTHAP), a comb hydrophobic associating polymer (CHAP) and a comb micro-block hydrophobic associating polymer (CBHAP), were comprehensively investigated. The experimental results proved that the thickening ability and salt resistance of the polymer was not significantly improved if only one intermolecular force functioned. On the contrary, multi-intermolecular forces, such as hydrophobic association and complexation, rendered a synergistic effect in the polymer solutions, through which the thickening property, salt resistance particularly $\mathrm{Ca}^{2+} / \mathrm{Mg}^{2+}$ resistance, and also the elasticity of the polymers were noticeably promoted. In oil displacement experiments, the EOR factors improved by CBHAP and CHAP can reach $27.1 \%$ and $18.8 \%$, which were much higher than that of KYPAM (11.5\%) and DTHAP (11.7\%).
\end{abstract}

\section{Introduction}

Supramolecular polymers refer to the polymeric materials formed via non-covalent interactions through self-assembly. ${ }^{1}$ These secondary forces may be $\pi-\pi$ interactions, ${ }^{2,3}$ hydrogen bonding ${ }^{4,5}$ or metal coordination, ${ }^{6}$ hydrophobic association, ${ }^{7,8}$ and electrostatic (ion pair of donor-acceptor) interactions. ${ }^{9}$ In aqueous solution, particularly in the presence of salt, hydrogen bonding, electrostatic interaction and hydrophobic association can be employed to form the self-assembling, improving the physical properties of water-soluble polymers.

Hydrogen bonding is an attractive electrostatic interaction between two polar molecules or within a polar molecule or group. For hydrogen bonding, the involved hydrogen $(\mathrm{H})$ is usually bonded to an electronegative atom such as nitrogen $(\mathrm{N})$, oxygen $(\mathrm{O})$ or fluorine $(\mathrm{F}) .^{10} \mathrm{In}$ a polyacrylamide solution, hydrogen bonding can result from the acylamino group. Nevertheless, this type of hydrogen bonding that is generated within the polymer molecule usually imposes a negative effect on the thickening ability for polyacrylamide. However, after the ethyoxyl (EO) groups are introduced into the polymer chains, the intermolecular hydrogen bonding could be established between the EO group and acylamide. Moreover, the size of the pendants can be easily modified by varying the

State Key Laboratory of Oil and Gas Reservoir Geology and Exploitation, Southwest Petroleum University, Chengdu 610500, Sichuan, China. E-mail: bwei@swpu.edu.cn number of EO groups. Based on this mechanism, a commercial anti-salt comb polymer, KYPAM, which also shows superior thickening capability to polyacrylamide, was successfully synthesized. PEG macromonomers produce comb-like polymers, which have PEG side chains and a hydrophobic backbone $\left(-\mathrm{CH}_{2}-\mathrm{CH}_{2}-\right)$ of EO groups, which can also significantly increase the viscosity of the polymer solution through the repulsion between the hydrophobic and hydrophilic groups. ${ }^{11,12}$ It has been known that complexation reactions would take place between multivalent metal ions and EO groups due to the lone pair of electrons provided by the oxygen of the oxyethylene group and thus fill the unoccupied orbital of metal ion, which in turn enhances the hydrophobicity of polar groups with polyether. ${ }^{13}$ Therefore, in saline water, multimolecular aggregation should promote the generation of a polymer solution via complexation and accordingly promote the $\mathrm{Ca}^{2+} / \mathrm{Mg}^{2+}$ resistance. Moreover, the multi-molecular aggregation dictates the hydrodynamic size of polymer molecules and also causes the solution viscosity to increase. In a salt solution, the hydrophobic association will be strengthened, which makes the hydrophobic associating polymers much more viscous compared to the conventional polymers. ${ }^{14}$ Unfortunately, thus far, the abovementioned polymers are not able to withstand the high degree of mineralization in saline water particularly having high content of $\mathrm{Ca}^{2+} / \mathrm{Mg}^{2+}$. Generally, the $\mathrm{Ca}^{2+} / \mathrm{Mg}^{2+}$ content for the abovementioned polymers is limited at $1000 \mathrm{mg} \mathrm{L}^{-1} .^{15,16}$ 
The salt resistance of a polymer can't be significantly improved if a single intermolecular force functions. On the contrary, it is hypothesized that by combining the abovementioned three intermolecular forces, the thickening capability and salt resistance of polymers would be noticeably promoted. Following this idea, EO groups and hydrophobic groups were incorporated in the polymer chains, and the multiintermolecular forces including hydrophobic association and complexation, are expected to be present and thus improve the thickening ability and salt resistance of the polymer. In this study, four types of polymers, a comb polymer (KYPAM), a hydrophobic associating polymer (DTHAP), a comb hydrophobic associating polymer (CHAP) and a comb micro-block hydrophobic associating polymer (CBHAP) were targeted to investigate the rheological properties of polymer solutions. Based on the features of these four polymers, the influences of chemical structure and multi-intermolecular forces could be distinguished. Overall, CBHAP, which contains hydrophobic association and complexation, exhibits advanced thickening capability, salt resistance, as well as elasticity compared with the other three polymers.

\section{Experimental}

\section{Materials}

2,2'-Azobis(2-methylpropionamidine)dihydrochloride (AIBA), acrylamide (AM), acrylic acid (AA), sodium dodecyl sulfate (SDS), $\mathrm{NaCl}, \mathrm{CaCl}_{2}$ and $\mathrm{MgCl}_{2}$ were purchased from Kelong Chemical Reagent Co. Partially hydrolyzed polyacrylamide (KYPAM, Mw 3000 million) was supplied by Beijing Hengju Chemical Group Co. Formation water was provided by Xin Jiang Oilfield, and the composition is given in Table 1. DTHAP $^{17}$ and $\mathrm{CBHAP}^{18}$ were prepared according to the reported procedures. The formulas of the polymers are showed in Table 2 and the chemical structures of three polymers were illustrated in Fig. 1.

\section{Rheological analysis}

Polymer stock solutions having concentration of $5000 \mathrm{mg} \mathrm{L}^{-1}$ were prepared and then placed in a water bath at $25{ }^{\circ} \mathrm{C}$ for $24 \mathrm{~h}$

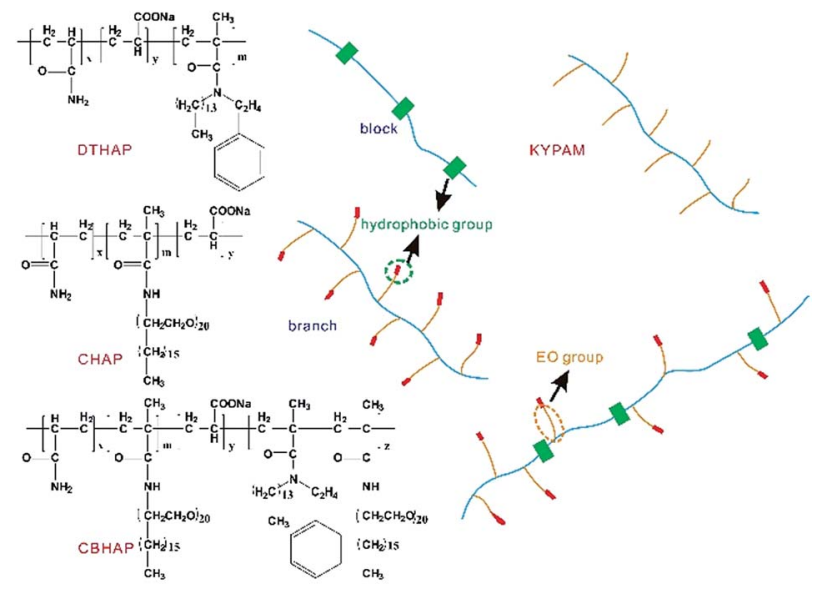

Fig. 1 Chemical structures of the evaluated polymers.

to ensure complete dissolution. The stock solutions were then diluted to specific concentrations. The viscometric measurements were carried out using a Brookfield ULTRA DV-III rotational viscometer at a shear rate of $7.34 \mathrm{~s}^{-1}$ at $25{ }^{\circ} \mathrm{C}$. The rheological study of CBHAP was performed at $50{ }^{\circ} \mathrm{C}$ using a MCR 302 rheometer (Anton Paar).

\section{FTIR and ${ }^{1} \mathrm{H}$-NMR characterization}

The FTIR and ${ }^{1} \mathrm{H}-\mathrm{NMR}$ of DTHAP and CBHAP were showed in the previous article. ${ }^{17,18}$ IR spectra of CHAP were measured with $\mathrm{KBr}$ pellets in a WQF-520 infrared spectroscopy (Beijing Reili Analytical Instrument). ${ }^{1} \mathrm{H}-\mathrm{NMR}$ spectrum was recorded on a Brucker AVANCE $400 \mathrm{MHz}$ spectrometer (Fig. 2).

\section{Microstructure characterization}

The aggregating morphology of CBHAP in aqueous and brine solutions was observed using an environmental scanning electron microscope (ESEM, Quanta 450, USA).

\section{Oil displacement experiment}

The experimental procedure was in accordance with that reported previously. ${ }^{17}$ In brief, the cores were flooded by water,

Table 1 Components of formation water

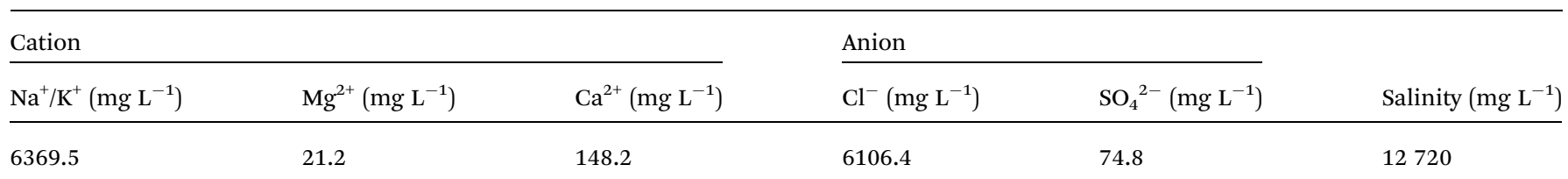

Table 2 Formulas of the polymers

\begin{tabular}{lllllll}
\hline Polymer & AM $(\mathrm{g})$ & AA $(\mathrm{g})$ & PETMAM $(\mathrm{g})$ & Acryloyl-O-20 $(\mathrm{g})$ & SDS $(\mathrm{g})$ & Structure \\
\hline DTHAP & 10 & 2.5 & 0.29 & 0 & 0.7 & Block \\
CHAP & 10 & 2.5 & 0 & 0.7 & 0 & Comb \\
CBHAP & 10 & 2.5 & 0.14 & 0.49 & 0.3
\end{tabular}




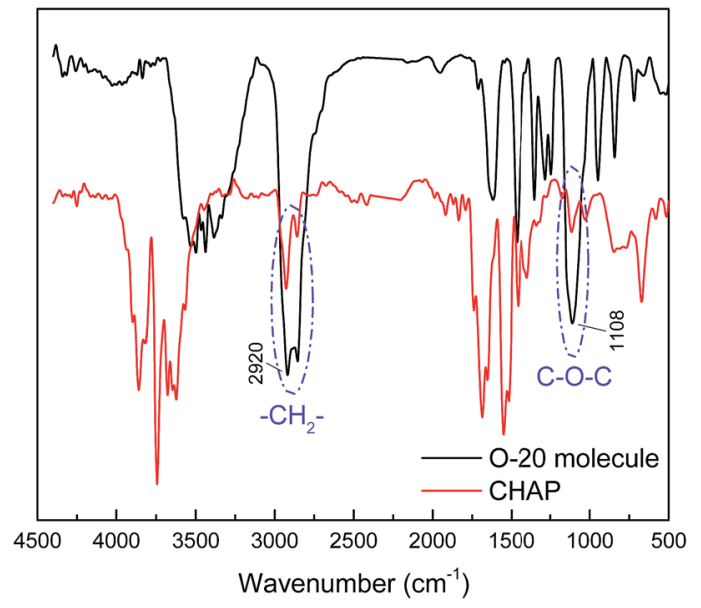

Fig. 2 The FTIR spectra of O-20 molecule and CHAP.

until the water cut reached 98\%, and then $0.3 \mathrm{PV}$ polymer solution was injected. After that, subsequent water flooding was conducted. In the experiments, the temperature was set to $30{ }^{\circ} \mathrm{C}$. In order to simulate the polymer flooding process at a high $\mathrm{Ca}^{2+} / \mathrm{Mg}^{2+}$ condition, the saline water was prepared with $5000 \mathrm{mg} \mathrm{L}^{-1} \mathrm{Ca}^{2+}$ and $500 \mathrm{mg} \mathrm{L}^{-1} \mathrm{Mg}^{2+}$ (Table 3).

\section{Stretch experiment}

The CBHAP was dissolved in the formation water. The solution was placed in a beaker at room temperature for $12 \mathrm{~h}$ to ensure full dissolution. Then, the CBHAP solution was injected into a bottle. The solution was slowly poured into an empty bottle. The distance of the two bottles was increased slowly until the break of CBHAP solution.

\section{Results and discussions}

In the curve of O-20 molecule, the band observed at $1109 \mathrm{~cm}^{-1}$ was attributed to the $\mathrm{C}-\mathrm{O}-\mathrm{C}$ and the band observed at 2920 $\mathrm{cm}^{-1}$ was attributed to the $-\mathrm{CH}_{2}-$. The two peaks can be found in the curve of CHAP. Therefore, the presence of the $\mathrm{O}-20$ molecule can be confirmed.

Fig. 3 presents the ${ }^{1} \mathrm{H}$ NMR spectrum of CHAP in $\mathrm{D}_{2} \mathrm{O}$. The characteristic peaks detected at 3.61-3.78 ppm, 3.36-3.46 ppm can be assigned to [- $\left.\mathrm{OCH}_{2} \mathrm{CH}_{2} \mathrm{O}-\right]$ of $\mathrm{O}-20$ molecule chain. The chemical shifts of 1.92-2.43 ppm and 1.28-1.83 ppm belong to the $-\mathrm{CH}$ and $-\mathrm{CH}_{2}$-protons in the polymer main chain, respectively. The signals at $1.23-1.28 \mathrm{ppm}$ and $0.93-0.97 \mathrm{ppm}$ belong to the $-\mathrm{CH}_{3}$ protons of $\left[\mathrm{CH}_{3} \mathrm{C}\right]$ and $\left[\mathrm{CH}_{3} \mathrm{CH}_{2}\right]$, respectively.

\section{Viscosifying property}

The thickening capability of the four polymers, KYPAM, DTHAP, CBHAP and CHAP, in fresh water at concentrations

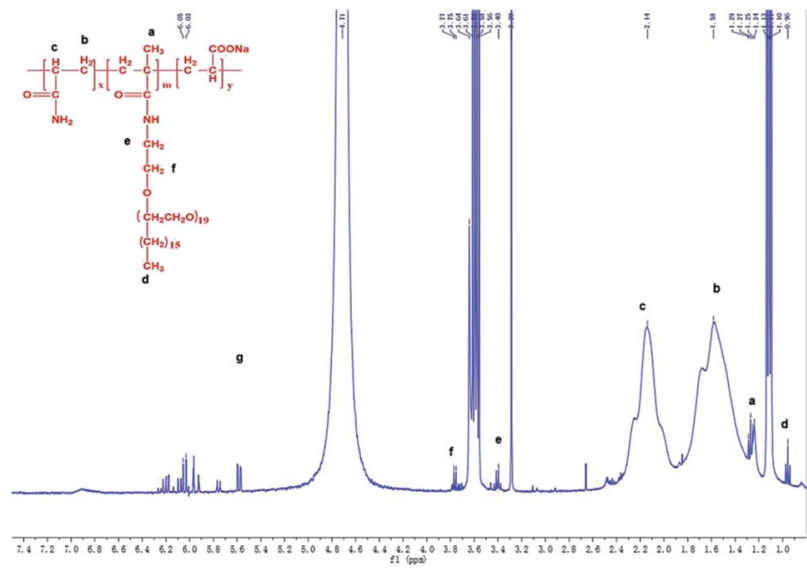

Fig. $3{ }^{1} \mathrm{H}-\mathrm{NMR}$ of CHAP.

ranging from $250 \mathrm{mg} \mathrm{L}^{-1}$ to $2000 \mathrm{mg} \mathrm{L}^{-1}$ was first investigated. Fig. 4 plots the apparent viscosity of the polymer solution as a function of concentration. Significant critical associating behavior was observed for three synthesized hydrophobic associating polymers, and the corresponding critical association concentration (CAC) was approximately 750 (DTHAP), 750 (CBHAP) and $1250 \mathrm{mg} \mathrm{L}^{-1}$ (CHAP), respectively. Increasing the concentration past the CAC, led to a rapid increase in the viscosity of the hydrophobic associating polymer. The commercial comb polymer, KYPAM, contained some EO groups that were randomly distributed in the branch chain. The repulsion between the EO group and acrylamide or carboxyl in KYPAM stretches the polymer chains and thus increases solution viscosity. Therefore, the thickening ability of KYPAM is usually better than that of HPAM under similar molecular weight and hydrolysis degree. The EO group, incorporated in CHAP, spaces the polymer backbone and hydrophobic group, and enables the intermolecular hydrophobic association, through which the thickening ability of CHAP is enhanced. Unlike CHAP, the hydrophobic groups in DTHAP are distributed in the polymer chains with a micro-block. As a result of the hydrophobic association, the viscosity of the DTHAP solution increased dramatically above its $\mathrm{CAC}, 750 \mathrm{mg} \mathrm{L}^{-1}$, and this value is considerably smaller than that for CHAP. In other words, the grafted hydrophobic group at the end of the EO group can enhance the thickening ability of the polymer; in addition, the micro-block structure can lower the CAC of the polymer. The combination of comb characteristics and the micro-block hydrophobic association appears to be an effective way to improve the viscous properties. Inspired by this, CBHAP, which has a comb and a micro-block structure, was successfully synthesized in this study. As anticipated, CBHAP possesses a lower CAC and prominent thickening capability. The viscosity of CBHAP is much higher than that of CHAP and DTHAP above

Table 3 The parameters of saline water and simulated oil

\begin{tabular}{llllllll}
\hline Saline water & $\mathrm{Na}^{+} \mathrm{mg} \mathrm{L}^{-1}$ & $\mathrm{Ca}^{2+} \mathrm{mg} \mathrm{L}^{-1}$ & $\mathrm{Mg}^{2+} \mathrm{mg} \mathrm{L}^{-1}$ & Salinity mg L & Oil & $\eta \mathrm{mPa} \mathrm{s}^{-1}$ & $\begin{array}{l}\text { Density } \mathrm{g} \mathrm{cm}^{-3} \\
{ }^{\circ}\end{array}$ \\
\hline 8142 & 5000 & 500 & 35854 & 11.85 & 8.2
\end{tabular}




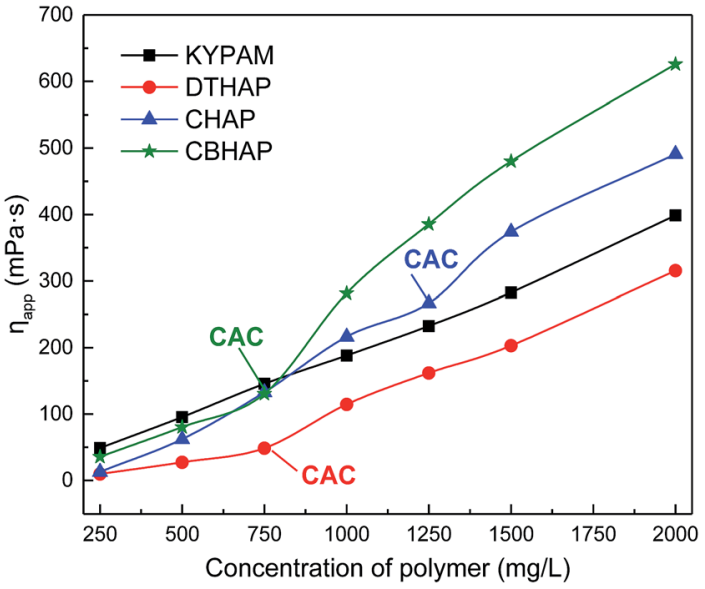

Fig. 4 The apparent viscosity of the polymer solution as a function of polymer concentration in fresh water.

$750 \mathrm{mg} \mathrm{L}{ }^{-1}$, suggesting the synergistic effect of the comb and the micro-block structure.

\section{Salt tolerance}

The salt-tolerance of the polymers was studied in this section. Formation water was used to dissolve the polymer powder. Fig. 5 depicts the viscous properties of each polymer. Herein, viscosity retention is defined as the viscosity ratio of the polymer in formation water to that in fresh water at an identical dosage. As shown in Fig. 5, when the viscosities of the four polymers are compared, it is clear that the viscosity of KYPAM in formation water is markedly lower than that of the other three synthesized polymers, suggesting that KYPAM is sensitive to salt. Therefore, it is believed that salt resistance has not been improved yet even when multi-intermolecular forces such as, hydrophobic group and hydrophilic group repulsion and hydrogen bonding between the EO group and acylaminde, are present. Similar to KYPAM, CHAP also presents a comb structure. However, the differentiation is that in CHAP the hydrophobic elements are loaded at the end of EO groups. The intermolecular force produced by hydrophobic association in CHAP results in a higher viscosity than that of KYPAM in formation water. Nevertheless, it was also found that there is an instability of the hydrophobic association because of EO group and hydrophobic group randomly distributed along main polymer chains that cause a lower viscosity retention compared
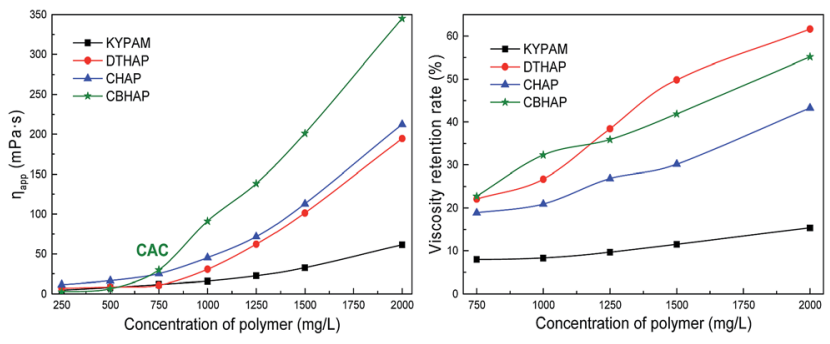

Fig. 5 The apparent viscosity of polymer in formation water. to DTHAP and CBHAP. The formation of multi-molecular aggregation results from the hydrophobic elements in the polymer chains with a micro-block, which renders the microblock hydrophobic associating polymer with an evident critical associating concentration. ${ }^{19}$ Hydrophobic groups in saline water spontaneously aggregate more easily than that in fresh water. Therefore, the viscosity retention rate (the ratio of the viscosity in saline water and the viscosity in fresh water) of DTHAP is higher than that of CHAP. As expected, CBHAP shows an advanced thickening ability in formation water, and the viscosity retention curve was located between DTHAP and CHAP. Therefore, to enhance polymer thickening ability, combining the comb polymer and the micro-block hydrophobic associating polymer appears to be an effective strategy.

In the presence of divalent cations such as $\mathrm{Ca}^{2+} / \mathrm{Mg}^{2+}$, multiintermolecular forces may be generated in aqueous solutions, including hydrophobic association and complexation, which lead to variations in viscosity response. For example, in DTHAP solutions, hydrophobic association is induced, whereas in CHAP solutions, weak hydrophobic association and complexation exist. In CBHAP solutions, the hydrophobic association becomes stronger compared to that in CHAP. Fig. 6 illustrates the viscosity response of the polymer solutions upon increasing the concentration of $\mathrm{Ca}^{2+} / \mathrm{Mg}^{2+}$. Overall, as the concentration of $\mathrm{Ca}^{2+} / \mathrm{Mg}^{2+}$ increases, the viscosity of CHAP and DTHAP steeply decreases; however, it was also observed that for DTHAP, a saltthickening behaviour was presented after $1000 \mathrm{mg} \mathrm{L}^{-1}$. Interestingly, CBHAP shows a positive viscous behaviour in $\mathrm{a}^{2+} /$ $\mathrm{Mg}^{2+}$ solution and also a higher viscosity retention than that of DTHAP and CHAP. It is believed that this result is caused by the strong hydrophobic association along with complexation.

In saline water, the chains of hydrophobic associating polymers would coil up due to the electrostatic shielding induced by the presence of cations. With the increasing cation content, electrostatic shielding will definitely cause intramolecular
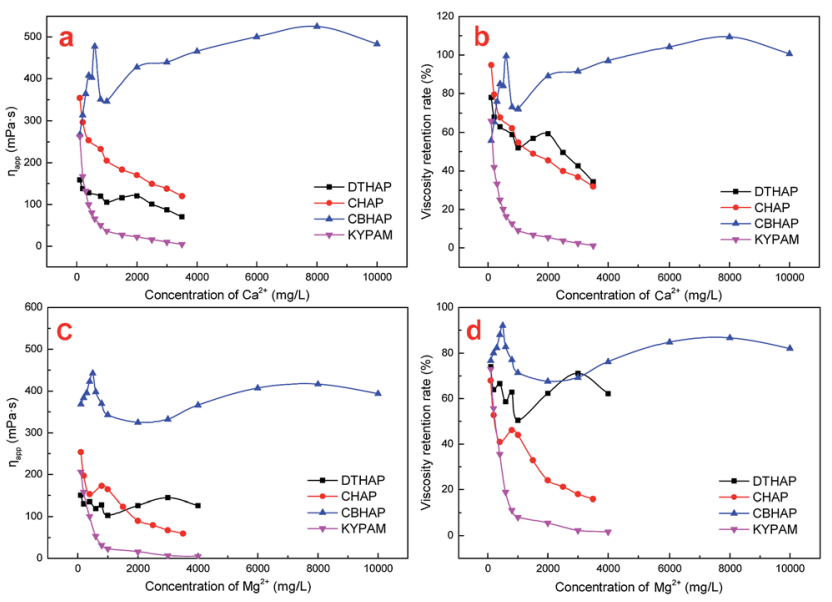

Fig. 6 Overall, as the concentration of $\mathrm{Ca}^{2+} / \mathrm{Mg}^{2+}$ increases, the viscosity of CHAP and DTHAP steeply decreases (a and c); interestingly, CBHAP shows a positive viscous behaviour in a $\mathrm{Ca}^{2+} / \mathrm{Mg}^{2+}$ solution and also a higher viscosity retention than that of DTHAP and CHAP (b and $d)$. 
hydrophobic association instead of intermolecular hydrophobic association and consequently the bulk viscosity of the polymer will significantly decreases. Nevertheless, EO groups broaden polymer chains and hydrophobic groups, and the intermolecular hydrophobic association is thus enhanced. Moreover, complexation formed by the EO groups is supposed to be able to hinder polymer chains coiling-up. ${ }^{\mathbf{1 8}}$ Therefore, the salt resistance of CBHAP is more prominent than that of DTHAP and CBHAP.

\section{Rheological properties}

Fig. 7 plots the shear viscosity of the polymers as a function of shear rate. It is indicated that CHAP, CBHAP and DTHAP show a pronounced shear-thinning behavior from intermediate to high shear rate. For CBHAP and DTHAP, a newtonian region was observed on the viscosity-shear rate curves at low shear rate. The critical shear rates (CSR) for CBHAP and DTHAP are 25.1 and $1 \mathrm{~s}^{-1}$, respectively.

In aqueous solution, the hydrophobic elements aggregate as clusters like surfactant micelles. A network formation can be generated due to the numerous 'anchor points' or 'physical junctions' in a solution and thus constrain the mobilization of the polymer chains, which in turn improves the thickening efficiency compared to non-associative polymers. ${ }^{20}$ Hydrophobic groups with double tails in DTHAP are physically crosslinked at low shear rate before $1 \mathrm{~s}^{-1}$, restraining the orientation along the shear direction. Multi-molecular forces further enhance the shear-resistance of CBHAP in formation water, which leads to significantly less viscosity loss than that of CHAP and DTHAP at increasing shear rates.

Since CBHAP shows the most advanced behaviour in thickening and salt-resistance relative to CHAP and DTHAP, the rheological properties of CBHAP in saline water were further investigated. Fig. 8 plots the shear viscosity of CBHAP solution as a function of shear rate at $50{ }^{\circ} \mathrm{C}$. A critical shear rate corresponding to the intersection on the curves, was observed. Before this critical shear rate, the shear viscosity of CBHAP in saline water was found to slightly increase with shear rate, suggesting a shear-thickening behaviour. With the increasing shear rate, the polymer chains are prone to stretch along the shear direction (Fig. 9), and thus the intermolecular forces including hydrophobic association and complexation, would be

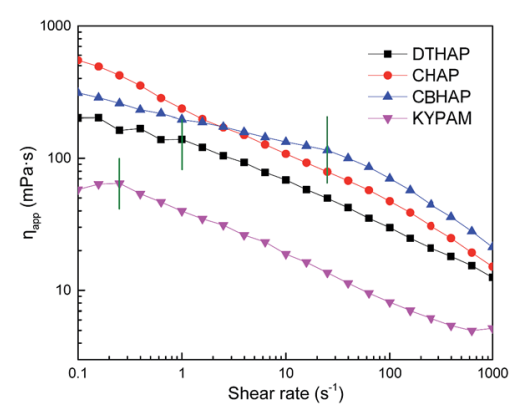

Fig. 7 Rheological curves of polymers $\left(1500 \mathrm{mg} \mathrm{L}^{-1}\right)$ in the formation water at $50{ }^{\circ} \mathrm{C}$.

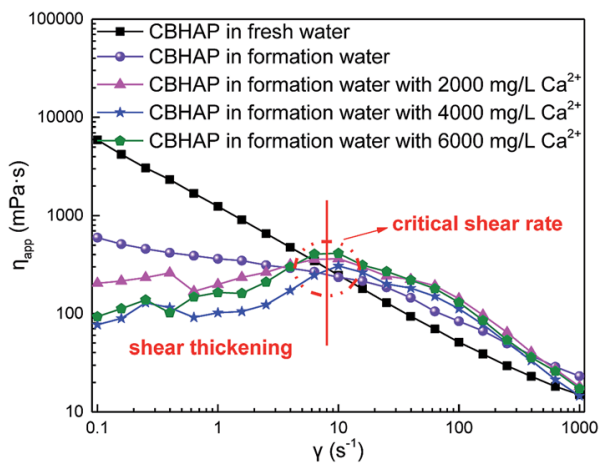

Fig. 8 Rheological curves of $\mathrm{CBHAP}\left(2000 \mathrm{mg} \mathrm{L}^{-1}\right)$ at $50^{\circ} \mathrm{C}$.

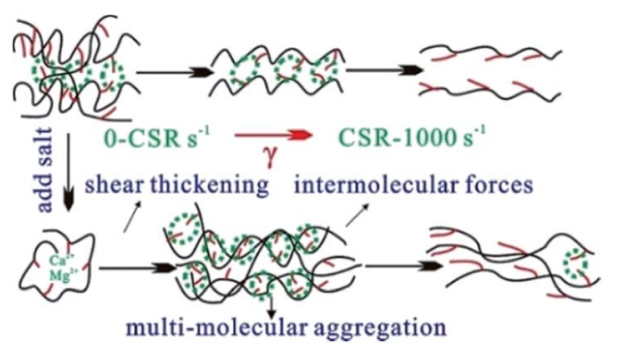

Fig. 9 Schematic of CBHAP in saline water under shearing.

enhanced. Beyond the critical shear rate, the viscosity of CBHAP in saline water outperforms that in fresh water. In addition, its viscosity gradually decreased with shear rate, which is a feature of a pseudoplastic fluid. Compared with viscosity curves in formation water containing $\mathrm{Ca}^{2+}$, in fresh water and formation water, only shear-thinning behavior was observed for CBHAP. This result might be caused by the intermolecular forces formed in the water containing $\mathrm{Ca}^{2+}$. As mentioned above, due to the presence of metal ions such as $\mathrm{Ca}^{2+}$, a complexation reaction would take place and the force is thus enhanced with an increase in $\mathrm{Ca}^{2+}$ concentration, which consequently allows the polymer to be resistant to shear and salt.

As can be seen from Fig. 10, CBHAP in formation water can be stretched above $20 \mathrm{~cm}$ in formation water. Different from melt spinning, CBHAP in formation water can be stretched into silk at room temperature without the necessity of heating to overcome the strong intermolecular forces; this shows a property similar to that of the hydrogels at a low concentration. ${ }^{21-23}$ In the system, the enhanced hydrophobic association due to salt, along with the complexation, enables the polymer solution

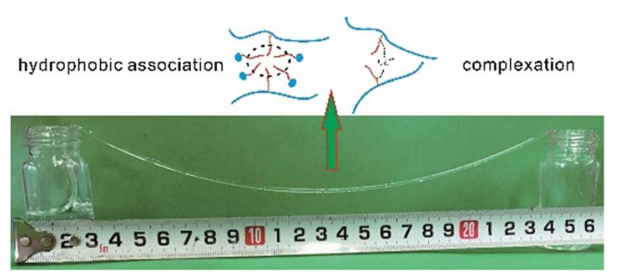

Fig. 10 Photograph of $\mathrm{CBHAP}\left(5000 \mathrm{mg} \mathrm{L}^{-1}\right)$ in formation water due to stretching. 
to be stretched into a filament, whereas traditional polymers, such as HPAM and KYPAM, will break quickly by stretching. The poor stretching properties of traditional polymers are attributed to the curled structure resulting due to the electrostatic shielding. The unique properties of the sol-gel transition of CBHAP will be studied next.

Plots of elastic $\left(G^{\prime}\right)$ and viscous $\left(G^{\prime \prime}\right)$ moduli of the polymers versus angular frequency are shown in Fig. 11. It indicates that the magnitudes of $G^{\prime}$ and $G^{\prime \prime}$ increase with frequency. It is also apparent that CBHAP possesses the greatest viscoelasticity relative to CHAP and DTHAP at low frequency. Electrostatic bridging, strong hydrophobic association and complexation play a synergistic role, which renders CBHAP's with excellent viscoelasticity. Therefore, a strong hydrophobic association is essential to improve the viscoelasticity of polymers.

\section{Environmental scanning electron microscope (SEM) images}

The microscopic feature of CBHAP was studied using SEM, as shown in Fig. 12. As seen, CBHAP in fresh water formed a multimolecular aggregation with space grid structure due to intermolecular forces. On the contrary, the structure of CBHAP in saline water was affected by electrostatic shielding and dehydration, forming a curled structure and a thinner polymer backbone. Thus it can be concluded that the viscosity of CBHAP in fresh water is greater than that in saline water. The result is
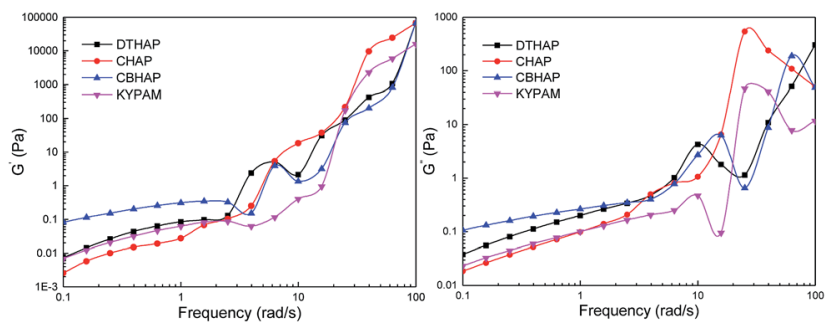

Fig. 11 Modulus of polymers in formation water.
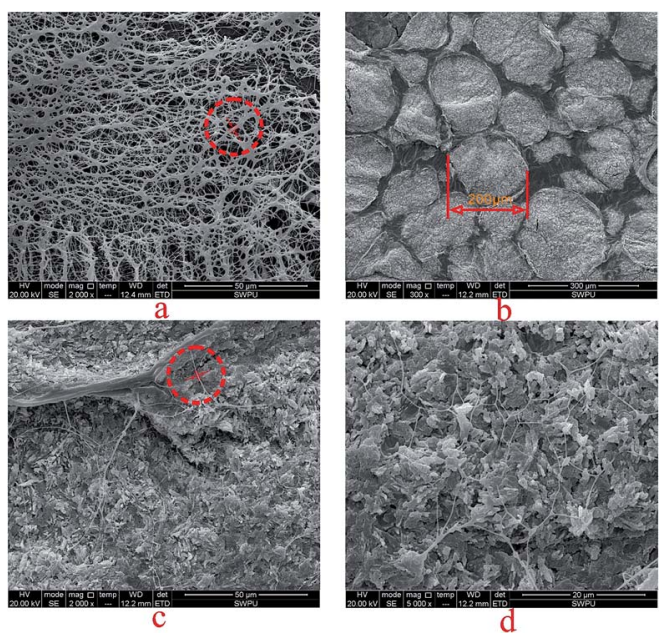

Fig. 12 SEM images of CBHAP (2000 $\mathrm{mg} \mathrm{L}^{-1}$ ): (a) in fresh water, $2000 \times$; (b) in formation water with $\mathrm{Ca}^{2+}, 300 \times$; (c) in formation water with $\mathrm{Ca}^{2+}, 2000 \times$; (d) in formation water with $\mathrm{Ca}^{2+}, 5000 \times$.
Table 4 The parameters of cores and results of the enhanced oil recovery of polymer flooding ${ }^{a}$

\begin{tabular}{llllll}
\hline Core & $D(\mathrm{~cm})$ & $L(\mathrm{~cm})$ & $V_{\mathrm{p}}(\mathrm{mL})$ & $K\left(\mu \mathrm{m}^{2}\right)$ & $V_{\mathrm{o}}(\mathrm{mL})$ \\
\hline 1 & 3.83 & 7.58 & 15.26 & 598.1 & 10.5 \\
2 & 3.84 & 7.59 & 13.2 & 840.2 & 9.0 \\
3 & 3.83 & 7.6 & 10.7 & 785.3 & 10.7 \\
4 & 3.83 & 7.61 & 14.53 & 725.6 & 10.1
\end{tabular}

${ }^{a} V_{\mathrm{p}}$, the pore volume of cores; $V_{\mathrm{o}}$, the volume of saturated oil; $C_{\mathrm{p}}$ : the concentration of polymers; $\eta$, the viscosity of polymer solution; $E_{1}$, the oil recovery ratio by water flooding; $E_{2}$, the total oil recovery ratio; $\mathrm{EOR}=E_{2}-E_{1}$.

\begin{tabular}{llllll}
\hline Polymer & $C_{\mathrm{p}}\left(\mathrm{mg} \mathrm{L}^{-1}\right)$ & $\eta(\mathrm{mPa} \mathrm{s})$ & $E_{1}(\%)$ & $E_{2}(\%)$ & EOR (\%) \\
\hline KYPAM & 2000 & 11.28 & 35.2 & 46.7 & 11.5 \\
DTHAP & 1800 & 60.54 & 39.4 & 51.1 & 11.7 \\
CHAP & 1350 & 50.69 & 39.1 & 57.9 & 18.8 \\
CBHAP & 1000 & 61.61 & 39.3 & 66.4 & 27.1
\end{tabular}

consistent with the rheological curves (Fig. 6) where the viscosity of CBHAP in saline water is lower than that in fresh water before critical shear rate.

\section{Enhanced oil recovery experiments}

The oil displacement experiments using the four polymers were conducted in this part. The data were summarized in Table 4. Fig. 13 and 14 present the displacement details. As already mentioned, KYPAM was very sensitive to the salt, resulting in a low viscosity. Even at $2000 \mathrm{mg} \mathrm{L}^{-1}$, the viscosity of KYPAM can only reach $11.28 \mathrm{mPa}$, which was lower than that of simulated oil. As a result, the improvement of oil recovery with KYPAM was much lower than that with CHAP and CBHAP. The oil recovery improved with DTHAP, but CHAP and CBHAP were different, even though the viscosities were similar, which is likely due to the difference in structure. The comb type structure gives a higher viscosity in which CBHAP at $1000 \mathrm{mg} \mathrm{L}^{-1}$ and CHAP at $1350 \mathrm{mg} \mathrm{L}^{-1}$ can reach a viscosity of about $60 \mathrm{mPa}$ s compared to DTHAP at $1800 \mathrm{mg} \mathrm{L}^{-1}$. Moreover, at a similar viscosity, the
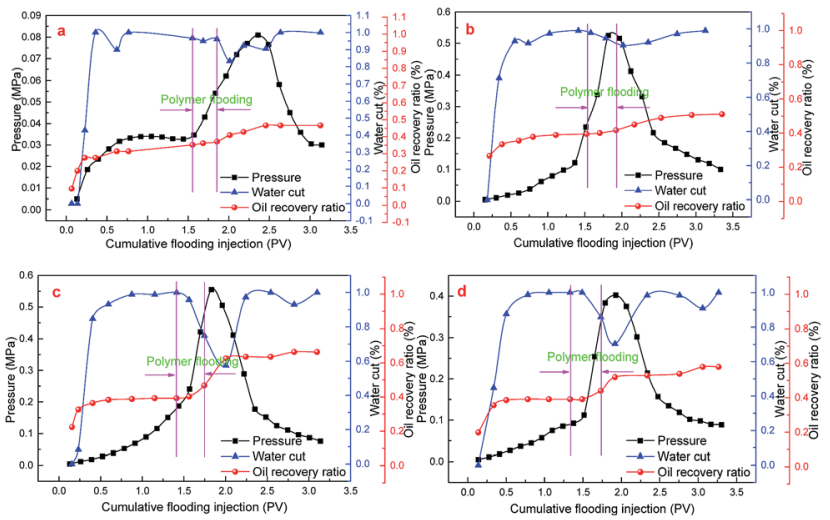

Fig. 13 The relationship of cumulative flooding injection and oil recovery as well as water cut: (a) KYPAM, (b) DTHAP, (c) CBHAP, (d) CHAP. 


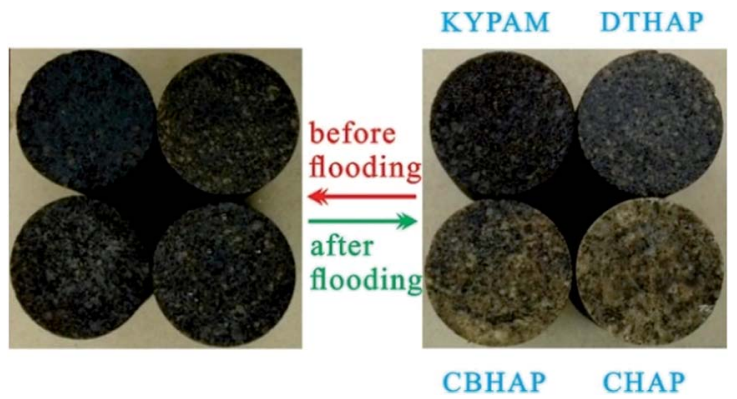

Fig. 14 The photograph of the cores.

enhanced oil recovery with CBHAP reached $27.1 \%$ and that with CHAP reached $18.8 \%$, which was much higher than that with DTHAP. Therefore, the comb type structure appears to be a good factor for enhancing oil recovery.

\section{Rheological property}

CBHAP in fresh water and saline water having concentration of $5000 \mathrm{mg} \mathrm{L}^{-1}$ was prepared and then placed in a water bath at $25{ }^{\circ} \mathrm{C}$ for $24 \mathrm{~h}$ to ensure complete dissolution. The solutions were then diluted to specific concentrations. The rheological study of CBHAP was performed at $50{ }^{\circ} \mathrm{C}$ using an MCR 302 rheometer (Anton Paar).

\section{Conclusion}

Four types of polymers with similar structures, including a commercial anti-salt comb polymer, KYPAM, and three synthesized functional polymers, DTHAP, CHAP and CBHAP, were investigated. Hydrophobic association and complexation are incorporated in the CBHAP solution with $\mathrm{Ca}^{2+} / \mathrm{Mg}^{2+}$. Three intermolecular forces provide a synergistic role, which significantly improve the thickening ability, salt resistance, particularly $\mathrm{Ca}^{2+} / \mathrm{Mg}^{2+}$ resistance, and elasticity of CBHAP. Via the intermolecular forces, CBHAP can be stretched above $20 \mathrm{~cm}$. In oil displacement experiments, the EOR values of CBHAP and CHAP can reach $27.1 \%$ and $18.8 \%$, which were much higher than that of KYPAM (11.5\%) and DTHAP (11.7\%). Therefore, using multi-molecular forces and comb type structures are an effective way to restrain coiling-up polymer chains and to improve the salt resistance and thickening ability, as well as the oil displacement property.

\section{Acknowledgements}

The authors wish to acknowledge the financial support received from the PetroChina Innovation Foundation (2015D-5006-
0212). Xinjiang Oilfield Company is also acknowledged for furnishing the formation water. The valuable comments made by the anonymous reviewers are also sincerely appreciated.

\section{Notes and references}

1 A. Jangizehi, S. R. Ghaffarian and E. Kowsari, J. Macromol. Sci., Phys., 2014, 53, 848-860.

2 X. Zhang, Y. Feng and S. Tang, Carbon, 2010, 48, 211-216.

3 A. Takai, M. Chkounda and A. Eggenspiller, J. Am. Chem. Soc., 2010, 132, 4477-4489.

4 G. F. De Paula, G. I. Netto and L. H. C. Mattoso, Polymers, 2011, 3, 928-941.

5 N. R. Gupta, P. P. Wadgaonkar and P. R. Rajamohanan, Carbohydr. Polym., 2015, 117, 331-338.

6 F. Wang, J. Zhang and X. Ding, Angew. Chem., 2010, 122, 1108-1112.

7 Q. Deng, H. Li and Y. Li, Aust. J. Chem., 2014, 67, 1396-1402.

8 B. Wei, L. Romero-Zerón and D. Rodrigue, Ind. Eng. Chem. Res., 2014, 53, 16600-16611.

9 F. Biedermann and O. A. Scherman, J. Phys. Chem. B, 2012, 116, 2842-2849.

10 C. Lin, T. Xiao and L. Y. Wang, Hydrogen-Bonded Supramolecular Polymers, Springer, 2015, vol. 87, pp. 321350.

11 J. N. Kizhakkedathu, J. Janzen and Y. Le, Langmuir, 2009, 25, 3794-3801.

12 P. D. Warren, D. V. McGrath and J. P. Vande Geest, Macromol. Mater. Eng., 2010, 295, 386-396.

13 T. Han, G. Xu and Y. Chen, J. Dispersion Sci. Technol., 2012, 33, 697-703.

14 X. Xin, L. Wang and J. Shen, J. Pet. Sci. Eng., 2014, 114, 15-21. 15 Y. F. Jia, W. L. Kang and L. W. Meng, Colloids Surf., A, 2014, 453, 117-124.

16 C. J. Zou, P. W. Zhao and X. Z. Hu, Energy Fuels, 2013, 27, 2827-2834.

17 F. Jiang, W. F. Pu and Y. B. Li, J. Appl. Polym. Sci., 2015, 132, 138-144.

18 W. Pu, F. Jiang and Y. He, RSC Adv., 2016, 6, 43634-43637.

19 A. Hill, F. Candau and J. Selb, Macromolecules, 1993, 26, 4521-4532.

20 P. A. Williams, Handbook of industrial water soluble polymers, John Wiley \& Sons, 2008.

21 K. Xu, H. An and C. Lu, Polymer, 2013, 54, 5665-5672.

22 Q. Wang, J. L. Mynar and M. Yoshida, Nature, 2010, 463, 339343.

23 T. Kakuta, Y. Takashima and M. Nakahata, Adv. Mater., 2013, 25, 2849-2853. 